\title{
CLASS NUMBERS AND IWASAWA INVARIANTS OF QUADRATIC FIELDS
}

\author{
JAMES S. KRAFT \\ (Communicated by William Adams)
}

\begin{abstract}
Let $\mathbf{Q}(\sqrt{-d})$ and $\mathbf{Q}(\sqrt{3 d})$ be quadratic fields with $d \equiv 2(\bmod$ $3)$ a positive integer. Let $\lambda^{-}, \lambda^{+}$be the respective Iwasawa $\lambda$-invariants of the cyclotomic $\mathbf{Z}_{3}$-extension of these fields. We show that if $\lambda^{-}=1$, then 3 does not divide the class number of $\mathbf{Q}(\sqrt{3 d})$ and $\lambda^{+}=0$.
\end{abstract}

\section{INTRODUCTION}

Let $k^{-}=\mathbf{Q}(\sqrt{-d})$ and $k^{+}=\mathbf{Q}(\sqrt{3 d})$ with $d$ a positive integer. In [5], Washington showed that constraints on the 3-Sylow subgroup and the fundamental unit of $k^{+}$force $\lambda^{-}$to be 1 , where $\lambda^{-}$is the Iwasawa $\lambda$-invariant associated to the cyclotomic $\mathbf{Z}_{3}$-extension of $k^{-}$. Here, using similar methods, we show that if $\lambda^{-}=1$ and 3 splits in $k^{-}$, then 3 does not divide the class number of $k^{+}$. Since recent results of Jochnowitz $[3,4]$ imply that there are infinitely many imaginary quadratic fields in which 3 splits and $\lambda^{-}=1$, we obtain as a corollary that there are infinitely many real quadratic fields $\mathbf{Q}(\sqrt{3 d})$ with 3 splitting in $\mathbf{Q}(\sqrt{-d})$ such that the class number of $\mathbf{Q}(\sqrt{3 d})$ is relatively prime to 3 . (We note that Horie [1] has proven a similar result concerning real quadratic fields by requiring that 3 neither divides the class number of nor splits in $\mathbf{Q}(\sqrt{-d})$.)

It then follows that there are infinitely many real quadratic fields with $\lambda^{+}=0$, where $\lambda^{+}$is the Iwasawa $\lambda$-invariant associated to the $\mathbf{Z}_{3}$-extension of $k^{+}$.

The author wishes to thank Lawrence Washington for many informative conversations.

We begin with a brief review of $p$-adic $L$-functions. For more details, see [6]. Let $p$ be an odd prime and let $\mathbf{Z}_{p}, \mathbf{Q}_{p}$ and $\mathbf{C}_{p}$ denote the $p$-adic integers, the $p$-adic rationals and the completion of the algebraic closure of $\mathbf{Q}_{p}$ respectively. Let $\omega$ denote the Teichmüller character and let $\psi$ be a primitive Dirichlet character of conductor $f$, with $p^{2}$ not dividing $f$. We let $d=f$ if $p$ does not divide $f$ and $d=$ $\frac{f}{p}$ if $p$ does divide $f$. The generalized Bernoulli number $B_{n, \psi}$ is defined by

$$
\sum_{a=1}^{f} \frac{\psi(a) e^{a t}}{e^{f t}-1}=\sum_{n=0}^{\infty} B_{n, \psi} \frac{t^{n}}{n !} .
$$

Received by the editors September 1, 1993 and, in revised form, August 1, 1994.

1991 Mathematics Subject Classification. Primary 11R11, 11R23, 11R29. 
The $p$-adic $L$-function $L_{p}(s, \psi)$ is the unique meromorphic $p$-adic function $\mathbf{Z}_{p} \rightarrow$ $\mathbf{C}_{p}$ which for $n \geq 1$ satisfies

$$
L_{p}(1-n, \psi)=-\left(1-\psi \omega^{-n}(p) p^{n-1}\right) \frac{B_{n, \psi \omega^{-n}}}{n} .
$$

In order to ensure that $L_{p}(s, \psi)$ is not identically zero, we now assume that $\psi$ is a non-trivial even character. If $O_{\psi}=\mathbf{Z}_{p}[\psi(1), \psi(2), \ldots]$, Iwasawa has shown that there is a power series $F(T, \psi) \in O_{\psi}[[T]]$ such that

$$
L_{p}(s, \psi)=F\left((1+p d)^{s}-1, \psi\right) .
$$

From the $p$-adic Weierstrass Preparation Theorem [6] we see that $F(T, \psi)=$ $G(T) U(T)$ where $U(T)$ is a unit of $O_{\psi}[[T]]$ and $G(T)$ is a distinguished polynomial. Then, $G(T)=a_{0}+a_{1} T+\cdots+a_{n-1} T^{\lambda-1}+T^{\lambda}$ and if $\pi$ generates the ideal of $O_{\psi}$ lying over $p$, then $\pi$ divides $a_{i}, 0 \leq i \leq \lambda-1$. We note that if $\psi$ is an even quadratic character and $p=3$, then $\lambda$ is related to the class group of certain number fields. That is, we let $k$ be the imaginary quadratic field associated to $\psi \omega^{-1}$ with $k_{\infty}$ its cyclotomic $\mathbf{Z}_{3}$-extension. Also, let $k_{n}$ be the unique subfield of $k_{\infty}$ of degree $3^{n}$ over $k$ and let $A_{n}$ be the 3-Sylow subgroup of $k_{n}$. Then, via the natural injection $A_{n} \rightarrow A_{n+1}$ for all $n \geq 0$,

$$
\bigcup_{n \geq 0} A_{n} \cong\left(\mathbf{Q}_{3} / \mathbf{Z}_{3}\right)^{\lambda}
$$

2

Let $K$ be a real quadratic field with character $\chi$, fundamental unit $\epsilon$, discriminant $D$ and class number $h^{+}$. Leopoldt's $p$-adic class number formula says that

$$
\frac{2 h^{+} \log _{p}(\epsilon)}{\sqrt{D}}\left(1-\frac{\chi(p)}{p}\right)=L_{p}(1, \chi)
$$

where $\log _{p}$ denotes the $p$-adic logarithm.

We now assume that $p=3$ and let $\lambda^{-}$(resp. $\lambda^{+}$) be the Iwasawa $\lambda$-invariant associated to the cyclotomic $\mathbf{Z}_{3}$-extension of $\mathbf{Q}(\sqrt{-d})$ (resp. $\mathbf{Q}(\sqrt{3 d})$ ) for the prime 3.

Theorem. Assume $d \equiv 2(\bmod 3)$ and $\lambda^{-}=1$. Then 3 does not divide the class number of $\mathbf{Q}(\sqrt{3 d})$. In particular, $\lambda^{+}=0$.

Proof. Let $\chi$ be the non-trivial even quadratic character of conductor $3 d$. Since 3 splits in $\mathbf{Q}(\sqrt{-d})$, we have that $L_{3}(0, \chi)=0$ from (1). Furthermore, since $\lambda^{-}=$ $1, F(T, \chi)=\left(b_{0}+b_{1} T\right) U(T)$ where $U(T)$ is a unit of $\mathbf{Z}_{3}[[T]]$ and $b_{1}$ is a 3 -adic unit. Because $L_{3}(0, \chi)=F(0, \chi)$, we have $F(T, \chi)=\left(b_{1} T\right) U(T)$. Since 3 does not divide $b_{1}, L_{3}(1, \chi) \not \equiv 0(\bmod 9)$. (See Lemma 1 of [5].) Then,

$$
\frac{2 h^{+} \log _{3}(\epsilon)}{\sqrt{D}} \not \equiv 0(\bmod 9)
$$

from (2). Thus, in order to prove that 3 does not divide $h^{+}$, it suffices to show that $\log _{3}(\epsilon) \equiv 0(\bmod 3 \sqrt{3 d})$. In order to prove this congruence, note that the 
3-integrality of $L_{3}(1, \chi)$ together with the fact that in this situation there is a $\sqrt{3 d}$ in the denominator of (2) imply that $\log _{3}(\epsilon)$ must have half-integral (non-integral) 3 -adic valuation. Thus, it is sufficient to show that $\log _{3}(\epsilon) \equiv 0 \bmod 3$. Let

$$
\epsilon=a+b \sqrt{3 d} \text { or } \frac{a+b \sqrt{3 d}}{2} \text {. }
$$

Then

$$
\epsilon^{2}-1 \equiv 2 a b \sqrt{3 d}(\bmod 3)
$$

Since

$$
\log _{3}\left(\epsilon^{2}\right)=\log _{3}\left(\epsilon^{2}-1+1\right) \equiv\left(\epsilon^{2}-1\right)-\frac{\left(\epsilon^{2}-1\right)^{2}}{2}+\frac{\left(\epsilon^{2}-1\right)^{3}}{3}(\bmod 3),
$$

we see that

$$
\log _{3}\left(\epsilon^{2}\right) \equiv 2 a b \sqrt{3 d}+8 a^{3} b^{3} d \sqrt{3 d}(\bmod 3) .
$$

Since $d \equiv 2(\bmod 3)$, we see that $\log _{3}\left(\epsilon^{2}\right) \equiv 0(\bmod 3)$. Thus, $\log _{3}(\epsilon) \equiv 0(\bmod 3)$ as well.

Finally, a theorem of Iwasawa [2] says that if 3 totally ramifies in the cyclotomic $\mathbf{Z}_{3}$-extension of $\mathbf{Q}(\sqrt{3 d})$ and $h^{+}$is not divisible by 3 , then $\lambda^{+}=0$.

In [4], Jochnowitz proves that given an arbitrary odd prime $p$, there are infinitely many imaginary quadratic fields in which $p$ splits and whose Iwasawa $\lambda$-invariant associated to $p$ equals 1 . This immediately implies the following.

Corollary. There are infinitely many real quadratic fields which have class number not divisible by 3 and whose Iwasawa $\lambda$-invariant associated to 3 equals zero.

Examples. We now give several examples. The first illustrates our theorem, the next two show that if $d \not \equiv 2(\bmod 3)$ and $\lambda^{-}=1$, then it is possible to have 3 dividing $h^{+}$, and the final one shows that if $d \equiv 2(\bmod 3)$ and $\lambda^{-} \neq 1$, then it is also possible to have 3 dividing $h^{+}$.

\begin{tabular}{|c|c|c|}
\hline$d$ & $\lambda^{-}$ & $h^{+}$ \\
\hline 23 & 1 & 1 \\
237 & 1 & 3 \\
262 & 1 & 6 \\
107 & 2 & 3 \\
\hline
\end{tabular}

\section{REFERENCES}

1. K. Horie, A note on basic Iwasawa $\lambda$-invariants of imaginary quadratic fields, Invent. Math. 88 (1987), 31-38. MR 88i: 11073

2. K. Iwasawa, A note on class numbers of algebraic number fields, Abh. Math. Sem. Univ. Hamburg 20 (1956), 257-258. MR 18:644d

3. N. Jochnowitz, A p-adic conjecture about derivatives of L-series attatched to modular forms, Proceedings of the Boston University Conference on $p$-Adic Monodromy and the $p$-Adic Birch and Swinnerton-Dyer Conjecture (to appear). CMP 94:13 
4. __ An alternative approach to non-vanishing theorems for coefficients of half integral weight forms mod $p$ and implications for Iwasawa's $\lambda$-invariant for quadratic fields (to appear).

5. L. Washington, Zeroes of p-adic L-functions, Sém Delange-Pisot- Poitou, Théorie des Nombres, 1980/1981, Birkhäuser, Boston, Basel, and Stuttgart, 1982. MR 84f: 12008

6. Introduction to cyclotomic fields, Graduate Texts in Math., Springer-Verlag, New York, 1982. MR 85g:11001

Department of Mathematics and Computer Science, Ithaca College, Ithaca, New YORK 14850

E-mail address: kraft@ithaca.edu 